\title{
Eficacia del ácido tranexámico para la disminución de la transfusión de sangre alogénica en la artroplastía total de cadera
}

\author{
Effectiveness of tranexamic acid for decreased allogenic blood transfusion in total hip arthroplasty \\ García-Dobarganes-Barlow FE, ${ }^{*}$ Romo-Aguilera IJ,${ }^{\ddagger}$ Negrete-Corona J,${ }^{\S}$ \\ Guevara Álvarez A, ${ }^{\S}$ Garcini-Munguía FA, ${ }^{\S}$ Téllez-Hoyos S\|
}

Hospital Ángeles de Querétaro.

RESUMEN. Introducción: La artroplastía total de cadera (ATC) puede causar una pérdida considerable de sangre. Para reducir la necesidad de transfusión de sangre se utilizan diversas técnicas preoperatorias como la administración de ácido tranexámico (ATX). Objetivo: Demostrar que el uso de ATX disminuye la necesidad de transfusión de sangre alogénica en pacientes operados de ATC. Material y métodos: estudio prospectivo, observacional, descriptivo, utilizado para observar la tasa de transfusión de sangre alogénica en pacientes a quienes se les practicó ATC primaria unilateral de Mayo de 2016 a Diciembre de 2017. A los pacientes incluidos en el estudio se les aplicó ATX preoperatoriamente, a las 24 horas se tomó una biometría hemática y se valoró la necesidad de transfusión sanguínea. Resultados: Se incluyó un total de 70 pacientes en el estudio. La $\mathrm{Hb}$ promedio postquirúrgica $11.7 \mathrm{mg} / \mathrm{dl}$ con un rango de 9.2 a $14.9 \mathrm{mg} / \mathrm{dl}$ y un Hto promedio de $37.1 \%$ con un rango de 30.2 a $44.2 \%$. Ninguno de los pacientes requirió de transfusión de sangre alogénica. Conclusiones: En este estudio se demuestra cómo el ATX es una estrategia transoperatoria para reducir la necesidad de transfusión de sangre alogénica en pacientes a quienes se realiza una ATC primaria.

Palabras clave: Artroplastía, cadera, transfusión, sangrado, ácido tranexámico.
ABSTRACT. Introduction: ATC can cause considerable blood loss. To reduce the need for blood transfusion, various preoperative techniques such as administration of tranexamic acid (ATX) are used. Objective: to demonstrate that the use of ATX decreases the need for allogeneic blood transfusion in patients operated on ATC. Material and methods: Prospective, observational, descriptive study, used to observe the allogeneic blood transfusion rate in patients who underwent unilateral primary ATC from May 2016 to December 2017. ATX was applied preoperatively and after 24 hours a blood count was taken and the need for blood transfusion was assessed. Results: A total of 70 patients were included in the study. The average postoperative $\mathrm{Hb} 11.7 \mathrm{mg} / \mathrm{dl}$ with a range of 9.2 to $14.9 \mathrm{mg} / \mathrm{dl}$ and an average $\mathrm{Ht}$ of $37.1 \%$ with a range of 30.2 to $44.2 \%$. None of the patients required allogeneic blood transfusión. Conclusions: This study demonstrates how ATX is a transoperative strategy to reduce the need for allogeneic blood transfusion in patients undergoing primary ATC.

Keywords: Arthroplasty, hip, transfusion, bleeding, tranexamic acid.

\section{Nivel de evidencia: IV}

* Jefe del Curso de Alta Especialidad en Cirugía Articular. Hospital Ángeles de Querétaro.

* Fellow del curso de Alta Especialidad en Cirugía Articular. Hospital Ángeles de Querétaro.

${ }^{\S}$ Profesor adjunto del Curso de Alta Especialidad en Cirugía Articular. Hospital Ángeles de Querétaro.

" Médico adscrito del Servicio de Anestesiología.

Dirección para correspondencia:

Dr. Fidel Enrique García Dobarganes Barlow

Jefe del Curso de Alta Especialidad en Cirugía Articular. Hospital Ángeles de Querétaro.

Teléfono: 4421923079

E-mail: fdobarganes@icloud.com. 


\section{Introducción}

La artroplastía total de cadera (ATC) es ampliamente reconocida como uno de los tratamientos quirúrgicos más eficaces para la artrosis de la cadera en etapa terminal. ${ }^{1}$ Más de 500,000 ATC se realizan cada año en Estados Unidos, ${ }^{2}$ representando aproximadamente un tercio de las artroplastías anuales realizadas. ${ }^{3}$ La necesidad de la ATC se ha incrementado y algunos estudios proyectan un aumento importante ${ }^{4}$ con una elevación a 572,000 en los Estados Unidos para el año $2030,{ }^{5}$ esto debido al aumento de la esperanza de vida, el envejecimiento de la población y la mejora del tratamiento médico perioperatorio. ${ }^{6}$ La ATC puede causar una pérdida considerable de sangre. La anemia postoperatoria a menudo retrasa la recuperación funcional y prolonga la estadía del paciente después de la artroplastía articular total, sometiendo a los pacientes a complicaciones iatrogénicas y a los hospitales a costos no deseados y de transfusiones de sangre. ${ }^{5}$ La transfusión de glóbulos rojos empaquetados tiene la ventaja de que restaura la capacidad de transporte de oxígeno y reemplaza el volumen intravascular; ${ }^{7}$ sin embargo, las transfusiones perioperatorias están asociadas a varios riesgos que incluyen la transmisión de agentes infecciosos, la reacción de transfusión hemolítica, la lesión pulmonar aguda relacionada con la transfusión, ${ }^{4}$ estancia hospitalaria ${ }^{4}$ prolongada y la mortalidad a corto plazo. Una tasa de transfusión hospitalaria de 20 a $40 \%$ para los procedimientos primarios de ATC es frecuente; sin embargo, existe una amplia variación en la práctica de transfusión con tasas que van de 4 a $80 \%{ }^{4}$ Para reducir la necesidad de transfusión de sangre se utilizan diversas técnicas preoperatorias como el cese de medicamentos asociados al sangrado junto con la evaluación y el tratamiento de la anemia preoperatoria. Entre las medidas intraoperatorias se incluyen hipotensión controlada, anestesia regional, hemostasia meticulosa, agentes hemostáticos y la administración de agentes antifibrinolíticos como el ácido tranexámico (ATX). ${ }^{8}$ Las medidas postoperatorias incluyen transfusión de sangre autóloga, flebotomía reducida, uso de vendajes compresivos y drenajes de reinfusión, quimioprofilaxis cautelosa para la trombosis venosa y la alteración de los factores desencadenantes de la transfusión.

El ATX es un derivado sintético del aminoácido lisina que produce actividad antifibrinolítica mediante la inhibición competitiva de los sitios de unión de la lisina en las moléculas de plasminógeno. A través de este proceso, se cree que la ATX es capaz de ayudar al cuerpo a retener los coágulos de sangre con mayor eficacia y por lo tanto, reducir la pérdida, ${ }^{9}$ en consecuencia, prevenir la disminución del nivel de hemoglobina $(\mathrm{Hb}){ }^{2}$ El ATX también desempeña un papel en los protocolos antiinflamatorios del reemplazo articular. ${ }^{1}$ La Asociación Americana de Cirujanos de Cadera y Rodilla, lba Academia Americana de Cirujanos Ortopédicos (AAOS), la Sociedad de Cadera, la Sociedad de Rodilla y la Sociedad Americana de Anestesia Regional y Medicina del Dolor han colaborado en el desarrollo de una guía de práctica clínica sobre el uso de AT en el reemplazo articular primario. ${ }^{3}$

Las intervenciones terapéuticas que combinan ventajas clínicas y fiscales son valiosas en una era en la que recortar los costos de atención médica se ha convertido en algo primordial. ${ }^{10}$ En los metaanálisis recientes de los ensayos controlados aleatorios se demostró que el efecto calculado estimado del ATX intravenoso (IV) sobre la pérdida total de sangre es de $305 \mathrm{ml}$ y una reducción en los requisitos de transfusión de sangre postoperatoria de $20 \%$ para ATX versus placebo ${ }^{11}$ en pacientes sometidos a ATC. Una de las desventajas teóricas del ATX es que puede haber un aumento en la probabilidad de formación de coágulos y esto puede presentar un riesgo adicional de TEV en pacientes que ya tienen múltiples factores de riesgo. ${ }^{12}$ Nuestra hipótesis es que el uso de ATX en pacientes operados de ATC reduce la necesidad de transfusión de sangre alogénica.

\section{Material y métodos}

Estudio prospectivo, observacional, descriptivo, utilizado para observar la tasa de transfusión de sangre alogénica en pacientes a quienes se les practicó ATC primaria unilateral de Mayo 2016 a Diciembre 2017.

Los criterios de inclusión fueron: pacientes de ambos géneros que fueron sometidos a una ATC primaria unilateral cementada o no cementada realizada por el mismo cirujano. Los criterios de exclusión fueron: pacientes operados de ATC bilateral o ATC de revisión, antecedente de trastornos de coagulopatías congénitas o adquiridas y/o tratamiento anticoagulante continuo, evento tromboembólico previo como trombosis venosa profunda (TVP) o tromboembolia pulmonar (TEP), falla renal o hepática, infarto al miocardio o enfermedad cerebrovascular y alergia al ATX. El criterio de eliminación fue por expediente incompleto.

A los pacientes incluidos en el estudio se les realizó radiografía anteroposterior y lateral de la cadera afectada, estudios de laboratorio consistentes en biometría hemática, hemotipo, química sanguínea básica, tiempos de coagulación, examen general de orina y examen dental; además, a los mayores de 45 años se les solicitó valoración por medicina interna para establecer el riesgo quirúrgico. El procedimiento fue realizado por el mismo cirujano. A todos los pacientes se les aplicó $1 \mathrm{~g}$ de ácido tranexámico intravenoso 10 minutos antes del procedimiento anestésico y una segunda dosis de $1 \mathrm{~g}$ ácido tranexámico intravenoso 10 minutos antes del cierre de la herida quirúrgica.

Con respecto a la cirugía, a todos los pacientes se les realizó abordaje posterior de cadera, se colocó prótesis total de cadera cementada o no cementada según la preferencia del cirujano, en todos los casos se colocó drenaje intraarticular de $1 / 8$ ". Se repararon los tejidos blandos con la técnica habitual y se colocaron apósitos estériles. El paciente en el postoperatorio recibió enoxaparina 40 mg subcutáneo cada 24 horas durante cuatro días, iniciando seis horas posteriores a la misma y posteriormente 
se continuó con rivaroxabán $10 \mathrm{mg}$ cada 24 horas por 28 días. Se tomó biometría hemática de control a las 24 horas del postoperatorio, se registraron los resultados y se valoró clínicamente al paciente; la decisión de realizar la transfusión sanguínea alógena se basó en los siguientes criterios: tener un hematocrito de $30 \%$ o menor y $\mathrm{Hb}$ $9.0 \mathrm{mg} / \mathrm{dl}$ o menor y clínicamente presentar datos de bajo gasto; estos criterios se establecieron en el grupo de trabajo de manera previa.

\section{Resultados}

Se incluyó un total de 70 pacientes en el estudio, de los cuales $53(75.71 \%)$ fueron diagnosticados con osteoartritis, $12(17.14 \%)$ con artritis reumatoide y cinco (7.14\%) con osteonecrosis. La edad promedio fue de 66 años con un rango de 22 a 86 años. El sexo predominante fue el femenino con $48(68.5 \%)$ pacientes y $22(31.42 \%)$ del sexo masculino. La $\mathrm{Hb}$ promedio prequirúrgica es de $14.8 \mathrm{mg} /$ dl con un rango de 12 a $17.5 \mathrm{mg} / \mathrm{dl}$ y el Hto promedio prequirúrgico es de $43.6 \%$ con un rango de 38.1 a $50.2 \%$. $\mathrm{La} \mathrm{Hb}$ promedio postquirúrgica $11.7 \mathrm{mg} / \mathrm{dl}$ con un rango de 9.2 a $14.9 \mathrm{mg} / \mathrm{dl}$ y un Hto promedio de $37.1 \%$ con un rango de 30.2 a $44.2 \%$. A ninguno de los pacientes se le indicó transfusión de sangre alogénica, debido a que ninguno presentó datos de bajo gasto o $\mathrm{Hb}$ menor de $9 \mathrm{mg} / \mathrm{dl}$ o Hto menor de $30 \%$. No se reportó ningún caso de TVP, TEP o alguna complicación durante el seguimiento que puede asociarse al uso del ATX y el tiempo de estancia hospitalaria fue de 36 horas en promedio, por lo que todos los pacientes fueron egresados sin complicaciones al día siguiente del procedimiento.

\section{Discusión}

Nuestro estudio muestra una disminución en la transfusión de sangre alogénica con base en nuestros criterios clínicos y de laboratorio. Históricamente, las tasas de transfusión para la ATC varían de $8 \%$ a casi $100 \%$ en las series informadas. Los riesgos de la transfusión incluyen la transmisión de la enfermedad (hepatitis B y C son las más comunes), reacciones de transfusión e inmunosupresión. Asimismo, los pacientes que reciben transfusiones de forma rutinaria tienen estancias hospitalarias más largas. ${ }^{13}$ En nuestro grupo de pacientes no aplicamos transfusión alguna, por lo cual se previno a nuestros pacientes de los riesgos señalados.

Como aminoácido sintético, el ATX es un inhibidor competitivo del plasminógeno y por lo tanto, actúa para disminuir la fibrinólisis. El trauma de la cirugía promueve la liberación de tPA (activador del plasminógeno tisular) y la fibrinólisis se activa. El proceso de fibrinólisis se inhibirá de forma natural 24 horas después de la cirugía. Pero los antifibrinolíticos (por ejemplo, el ATX) pueden bloquear el proceso de activación (plasminógeno a plasmina) en una etapa más temprana y por lo tanto, reducir la pérdida de sangre después de la operación, ${ }^{14}$ aspecto que no fue contemplado en este trabajo.

Consideramos que el ATX ha demostrado limitar la pérdida de sangre postoperatoria y la necesidad de transfusión de sangre alogénica. Como resultado, en nuestro grupo de trabajo se ha convertido en una herramienta de uso común para el manejo del sangrado después de la ATC. ${ }^{3}$ Aunque la eficacia de la ATX ha sido bien documentada con numerosos ensayos clínicos aleatorizados y metaanálisis posteriores, sospechamos que muchos anestesiólogos y cirujanos ortopédicos siguen teniendo inquietudes con respecto a la seguridad de su aplicación.

\section{Conclusiones}

En este estudio se demostró que el ATX es una estrategia transoperatoria que disminuye la necesidad de transfusión de sangre alogénica en pacientes operados de ATC primaria.

\section{Bibliografía}

1. Lei Y, Huang Q, Huang Z, Xie J, Chen G, Pei F. Multiple-dose intravenous tranexamic acid further reduces hidden blood loss after total hip arthroplasty: a randomized controlled trial. J Arthroplasty. 2018; 33(9): 2940-5. Available from: https://doi.org/10.1016/j. arth.2018.04.024.

2. Luo Z, Wang H, Wang D, Zhou K, Pei F, Zhou Z. Oral vs intravenous vs topical tranexamic acid in primary hip arthroplasty: a prospective, randomized, double-blind, controlled study. J Arthroplasty. 2018; 33(3): 786-93. Available from: https://doi.org/10.1016/j. arth.2017.09.062.

3. Fillingham YA, Ramkumar DB, Jevsevar DS, Yates AJ, Shores $\mathrm{P}$, Mullen K, et al. The efficacy of tranexamic acid in total hip arthroplasty: a network meta-analysis. J Arthroplasty. 2018; 33(10): 3083-9.e4. Available from: https://doi.org/10.1016/j. arth.2018.06.023.

4. Churchill JL, Puca KE, Meyer ES, Carleton MC, Truchan SL, Anderson MJ. Comparison of $\varepsilon$-aminocaproic acid and tranexamic acid in reducing postoperative transfusions in total hip arthroplasty. $J$ Arthroplasty. 2016; 31(12): 2795-9.e1. Available from: http://dx.doi. org/10.1016/j.arth.2016.05.011

5. North WT, Mehran N, Davis JJ, Silverton CD, Weir RM, Laker MW. Topical vs intravenous tranexamic acid in primary total hip arthroplasty: a double-blind, randomized controlled trial. $J$ Arthroplasty. 2016; 31(5): 1022-6. Available from: http://dx.doi. org/10.1016/j.arth.2015.11.003.

6. Park KJ, Couch CG, Edwards PK, Siegel ER, Mears SC, Barnes CL. Tranexamic acid reduces blood transfusions in revision total hip arthroplasty. J Arthroplasty. 2016; 31(12): 2850-5.e1. Available from: http://dx.doi.org/10.1016/j.arth.2016.05.058.

7. Demos HA, Lin ZX, Bar WR, Wilson SH, Robertson DC, Jr VDP. Process improvement project using tranexamic acid is cost-effective in reducing blood loss and transfusions after total hip and total knee arthroplasty. J Arthroplasty. 2017; 32(8): 2375-80.

8. Wei W, Wei B. Comparison of topical and intravenous tranexamic acid on blood loss and transfusion rates in total hip arthroplasty. $J$ Arthroplasty. 2014; 29(11): 2113-6. Available from: http://dx.doi. org/10.1016/j.arth.2014.07.019.

9. Tuttle JR, Ritterman SA, Cassidy DB, Anazonwu WA, Froehlich JA, Rubin LE. Cost bene fi t analysis of topical tranexamic acid in primary total hip and knee arthroplasty. J Arthroplasty. 2014; 29(8): 1512-5. Available from: http://dx.doi.org/10.1016/j. arth.2014.01.031. 
10. Whiting DR, Duncan CM, Sierra RJ, Smith HM. Tranexamic acid bene fi ts total joint arthroplasty patients regardless of preoperative hemoglobin value. J Arthroplasty. 2015; 30(12): 2098-101. Available from: http://dx.doi.org/10.1016/j.arth.2015.05.050.

11. Madsen R V, Nielsen CS, Kallemose T, Husted H, Troelsen A. Low risk of thromboembolic events after routine administration of tranexamic acid in hip and knee arthroplasty. $J$ Arthroplasty. 2017; 32(4): 1298-303. Available from: http://dx.doi.org/10.1016/j. arth.2016.10.015.

12. Trial C, Stowers MDJ, Aoina J, Vane A, Poutawera V, Hill AG, et al. Tranexamic acid in knee surgery study-a multicentered, randomized, controlled trial. J Arthroplasty. 2017; 32(11): 3379-84. Available from: https://doi.org/10.1016/j.arth.2017.05.058.

13. Benjamin JB, Colgan KM. Are routine blood salvage/preservation measures justified in all patients undergoing primary TKA and THA? J Arthroplasty. 2015; 30(6): 955-8. Available from: http://dx.doi. org/10.1016/j.arth.2015.01.031.

14. Rajesparan K, Biant LC, Ahmad M, Field RE. The effect of an intravenous bolus of tranexamic acid on blood loss in total hip replacement. J Bone Jt Surg Br. 2009; 91(6): 776-83. Available from: http://www.bjj.boneandjoint.org.uk/cgi/doi/10.1302/0301620X.91B6.22393. 